\title{
An approach to prioritize the regression test cases of object-oriented programs
}

\author{
Chhabi Rani Panigrahi $\cdot$ Rajib Mall
}

Received: 12 August 2012/Accepted: 14 March 2013/Published online: 10 April 2013

(C) CSI Publications 2013

\begin{abstract}
We propose a regression test case prioritization technique for object-oriented programs. We first construct an intermediate graph model of a program from its source code. When the program is modified, the model is updated to reflect the changes. Our constructed model represents control and data dependencies, and information pertaining to various types of dependencies arising from object-relations such as association, inheritance and aggregation. We determine the affected nodes in the model by constructing the union of the forward slices corresponding to each changed model element. A test case that covers a larger number of affected model elements is given higher priority. Our experimental results indicate that our approach on an average achieves an increase in the APFD metric value by $25.70 \%$ as compared to a related approach.
\end{abstract}

Keywords Software maintenance - Regression testing · Regression test case prioritization · Slicing

\section{Introduction}

Regression testing involves rerunning relevant test cases from existing test suite to build confidence that there is no unintended side-effects due to changes made to a software. A naive approach would be to run the entire test suite after every change. This is usually costly, wasteful of resources, and requires unduly long time. To overcome this problem a

C. R. Panigrahi $(\bowtie) \cdot$ R. Mall

Department of Computer Science \& Engineering, Indian Institute of Technology Kharagpur, Kharagpur, India

e-mail: panigrahichhabi@gmail.com; chhabi@cse.iitkgp.ernet.in

R. Mall

e-mail: rajib@cse.iitkgp.ernet.in large number of regression test selection techniques have been proposed [1-3]. However, regression test selection techniques often select significant number of test cases even for small changes made to a program. Researchers have attempted to make regression testing more effective by developing various approaches such as test suite minimization (TSM) [4-7] and test case prioritization (TCP) [8, $9,10,11]$. These techniques usually target to reduce the cost or improve the effectiveness of regression testing.

Regression TCP techniques provide a method to order test cases according to certain criteria, so that the highest priority test cases can be executed earlier in a regression testing session. Further, when it is possible to execute only a few test cases, the most fault-revealing test cases can be executed. TCP techniques usually target to order test cases based on the rate of fault detection or the rate of code coverage $[8,9]$. Most of the TCP techniques proposed in the literature were developed in the context of procedural programs. These existing TCP techniques do not work satisfactorily [8-10] for object-oriented programs, because these do not consider the implicit dependencies that arise due to object-relations.

Binder pointed out that in object-oriented programs methods tend to be small and simple [12]. Therefore, the programming complexities move from intra-procedural to inter-procedural aspects. Therefore, it can be assumed that consideration of dependencies arising on account of objectrelations in regression test prioritization is important. In this context, we have used a program model that represents the object-relations in an object-oriented program in addition to the traditional program dependencies. Again a test case which covers higher number of affected nodes in the dependency model has a higher chance to detect an error(s) than a test case which covers less number of affected nodes. Based on the above fact, we propose a 
regression TCP technique that prioritizes test cases according to the number of affected nodes covered by a test case in the dependency model of an object-oriented program.

This paper is organized as follows: In Sect. 2, we discuss certain background concepts used in our work. Review of the related regression TCP techniques is presented in Sect. 3 and our regression TCP technique is described in Sect. 4 In Sect. 5, we describe the experimental study carried out by us to prove the effectiveness of our approach. In Sect. 6, we present a comparison of our technique with related work and finally conclude the paper in Sect. 7.

\section{Basic concepts}

In this section we describe some basic concepts that form the basis of our work.

\subsection{SDG of object-oriented programs}

System dependence graph (SDG) was first introduced by Horwitz et al. [13] and was used to model procedural programs. Later on, SDG was extended by Larsen and Harrold to model object-oriented programs [14] and is named as extended system dependency graph (ESDG).

An ESDG is a directed, connected graph $G=(V, E)$, consisting of a set $V$ of vertices and a set $E$ of edges. In the following, we describe the different types of vertices and edges in an ESDG.

\subsubsection{ESDG vertices}

- Statement vertices: Program statements that are present in the body of the methods are represented by statement vertices. There are two types of statement vertices: simple statement vertices and call vertices. Method call statements (call sites) are represented by call vertices, whereas all other program statements such as assignments, loops and conditionals are represented by simple statement vertices.

- Entry vertices: In an ESDG, classes and methods have entry vertices. A class entry vertex represents an entry into a class and a method entry vertex represents an entry into a method.

- Parameter vertices: These are used to represent parameter passing between a caller and a callee method. The parameter vertices are of four types. These include formal-in, formal-out, actual-in, and actual-out. The actual-in and actual-out vertices are created for each call vertex and formal-in and formalout vertices are created for each method entry vertex.
- Polymorphic choice vertex: This vertex is used to represent dynamic choice among the possible bindings in a polymorphic call.

\subsubsection{ESDG edges}

- Data dependence edge: Data dependence edges are used to represent the data dependence relations existing among different statement vertices.

- Control dependence edge: A control dependence edge is used to represent control dependence relations between two statement vertices.

- Call edge: A call edge is used to connect a call site to a method entry vertex and also to connect various possible polymorphic method call vertices to a polymorphic choice vertex.

- Parameter dependence edge: Parameter dependence edges are used for passing values between actual and formal parameters in a method call. Parameter dependence edges are of two types: parameter-in and parameter-out edges.

- Summary edge: A summary edge represents the transitive dependence between actual-in and actual-out vertices.

- Class member edge: A class member edge is used to represent the membership relation between a class and its methods. A class entry vertex is connected to a method entry vertex by using a class member edge.

A class is represented in an ESDG by a class dependence graph (ClDG) [3]. Each method in a CIDG is represented by a procedure dependence graph [15]. The root node of a CIDG is represented by a class entry vertex. Each method in a class is associated with a method entry vertex. The class entry vertex is connected to the method entry vertex for each method in a class by class member edges. In a ClDG, method calls are represented by call vertices. A formal-in vertex is added for each formal parameter in the method, and one formal-out vertex for each formal reference parameter that is modified by the called method.

The methods of a class can interact among each other or with methods of other classes. In a CIDG, a method call is represented by a call vertex. For each method call vertex, the actual-in and actual-out vertices as well as formal-in and formal-out vertices are added for each called method. The actual-in parameter vertices are connected to the corresponding formal-in vertices in the called method by parameter-in edges. The formal-out vertex of the called method is connected to the corresponding actual-out vertex at the calling method by a parameter-out edge. A CIDG represents effects of return statements by connecting each return statement to its corresponding call vertex using a parameter-out edge. Similarly, for the actual-in parameters 
that may affect the returned value, summary edges are added between the actual-in vertices and the actual-out vertex. For a derived class, the representation of the base class method is reused for representing the inherited methods. Apart from connecting the class entry vertex of a class to its method entry vertices of locally defined methods, class member edges are constructed to connect the class entry vertex of the derived class to the method entry vertices of the methods inherited by the derived class.

In an object-oriented program, a class may instantiate another class. When a class $C_{1}$ instantiates another class $C_{2}$, an implicit call to the constructor of $C_{2}$ is made. To represent instantiation of a class $C_{2}$ by $C_{1}$ in ESDG, a call vertex is created in $C_{1}$. A call edge connects this call vertex to the constructor of $C_{2}$. When a method $m_{1}$ in a class $C_{1}$ calls a public method $m_{2}$ of another class $C_{2}$, the call vertex in $C_{1}$ is connected to the entry vertex of $m_{2}$ by a call edge.

An ESDG can also represent polymorphic relationships between a calling method and its called methods. A polymorphic method call in an object-oriented program implies that the destination of a method call is determined at runtime. An ESDG uses a polymorphic choice vertex to represent the dynamic choice among the possible destinations. The polymorphic choice vertex has call edges that are connected to the subgraphs representing calls to each possible destinations. Figure 2 shows the partial ESDG representation of the class Coinbox for the Java code as given in Fig. 1. As shown in Fig. 2, class entry node of Coinbox class is connected to all the method entry nodes by class member edges. For readability sake, we have not shown all the edges in the ESDG.

\subsection{Regression testing cycle}

After a software is released, the failure reports and change requests from the user are accumulated using a defect tracking system [16]. At suitable intervals the program is modified to address all change requests and failure reports. After the required changes have been carried out, regression testing is carried out to revalidate the affected parts of the code. The failures identified by the regression testing are fixed and this cycle is repeated until there are no more regression test failures. Each cycle of regression testing and the fixing of identified bugs is referred to as a regression testing cycle.

The activities undertaken during a regression testing cycle include test setup, test selection, test prioritization, test case execution and failure report preparation. After regression testing are successfully completed, a new version of the software is released, which then undergoes similar regression testing cycles. Please note that, there can be multiple regression testing cycles before each release of a software.

\subsection{Program slicing}

The concept of a program slice was first introduced by Weiser to aid debugging of programs [17]. Since then a significant amount of research results on code slicing have been reported in the literature [18]. Code slicing techniques have been used in various application areas such as program debugging, testing, program understanding, and software maintenance $[13,19]$. A program slice consists of all those program statements that can potentially affect the values computed at some point of interest called the slicing criterion [13, 14]. For different applications, different types of program slices have been proposed [18]. A backward program slice at a program point $p$ with respect to a variable $v$ contains all statements in the program, including conditionals, that might affect the value of $v$ at $p$ [13], whereas a forward program slice at a program point $p$ with respect to a variable $v$ contains all statements in the program, including conditionals, that might be affected by any modifications to $v$ at $p$ [13].

Slice is calculated as a graph-reachability problem in the dependence graph of a program. The inter-procedural slicing algorithm was first proposed by Horwitz et al. [13]. Later on, Horwitz et al.'s algorithm was extended by Larsen and Harrold [14] for slicing on a dependence graph of an object-oriented software. A comprehensive survey on program slicing techniques has been reported in [20].

\subsection{APFD metric}

To measure the average rate of fault detection of a regression test suite the average percentage of faults detected (APFD) metric was proposed by Rothermel et al. [21]. The APFD metric has been used by several researchers $[22,23]$ to evaluate the effectiveness of a test prioritization scheme. The APFD metric for a test suite is calculated by taking the weighted average of the number of faults detected during execution of the program with the test suite. APFD metric values range from 0 to 100 and a higher number indicates a faster rate of fault detection.

The APFD metric can be calculated using the following expression. Let $T$ be the original test suite containing $n$ test cases, and let $F$ be a set of $m$ faults revealed by $T$. Let $T^{\prime}$ be an ordering of $T$. In $T^{\prime}$, let $T F_{i}$ be the first test case which reveals a fault $i$. Then the APFD metric for test suite $T^{\prime}$ can be obtained by using the equation:

$A P F D=1-\frac{T F_{1}+T F_{2}+\ldots \ldots+T F_{n}}{n * m}-\frac{1}{2 n}$

For example, consider a program with a test suite of five test cases, that have been labeled $a$ through $e$. Suppose 
Fig. 1 Java code of the class Coinbox

Fig. 2 A partial ESDG representation of the class Coinbox as given in Fig. 1

\begin{tabular}{|c|c|c|}
\hline CE1: & class Coinbox \{ & S16: d1. dispense (selection); $\}$ \\
\hline E2: & public Coinbox(unsigned flag) \{ & \} \\
\hline S3: & totCoins $=$ curCoins $=$ alVend $=0$; & E17: public unsigned isalvend ( ) \{ \\
\hline S4: & checkflag = flag; $\}$ & S18: $\quad$ return alVend; $\}$ \\
\hline E5. & nublic void insert () f & E19: public void return ( ) \{ \\
\hline 56. & curCoins ++; & S20: $\quad$ c1. retcoins (curCoins); \\
\hline $\mathrm{C}$ & if (curCoins $>1$ ) & curCoins = 0 \\
\hline S): & & S22: $\quad$ alVend $=0 ; \quad\}$ \\
\hline S8: & alVend = 1 ; & S23: if (checkflag) \\
\hline S9: & if (checkflag) & $\operatorname{check}()$ \\
\hline S10: & $\operatorname{check}()$ & 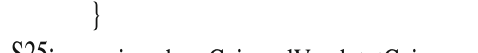 \\
\hline & 1 & unsigned curCoins, alVend, totCoins ; \\
\hline & \} & Dispenser $\mathrm{d} 1$; \\
\hline E11: & public void vend (unsigned selection) \{ & Coinreturn cl; \\
\hline S12: & if (isalvend) \{ & public void check( ) \{ \\
\hline S13: & totCoins $+=$ curCoins & if $($ (curCoins <2) \&\& ( alVend $))$ \\
\hline S14: & curCoins $=0$; & S30: $\quad$ println ( "User can vend for free"); \\
\hline S15: & alVend = 1; & \} \\
\hline
\end{tabular}

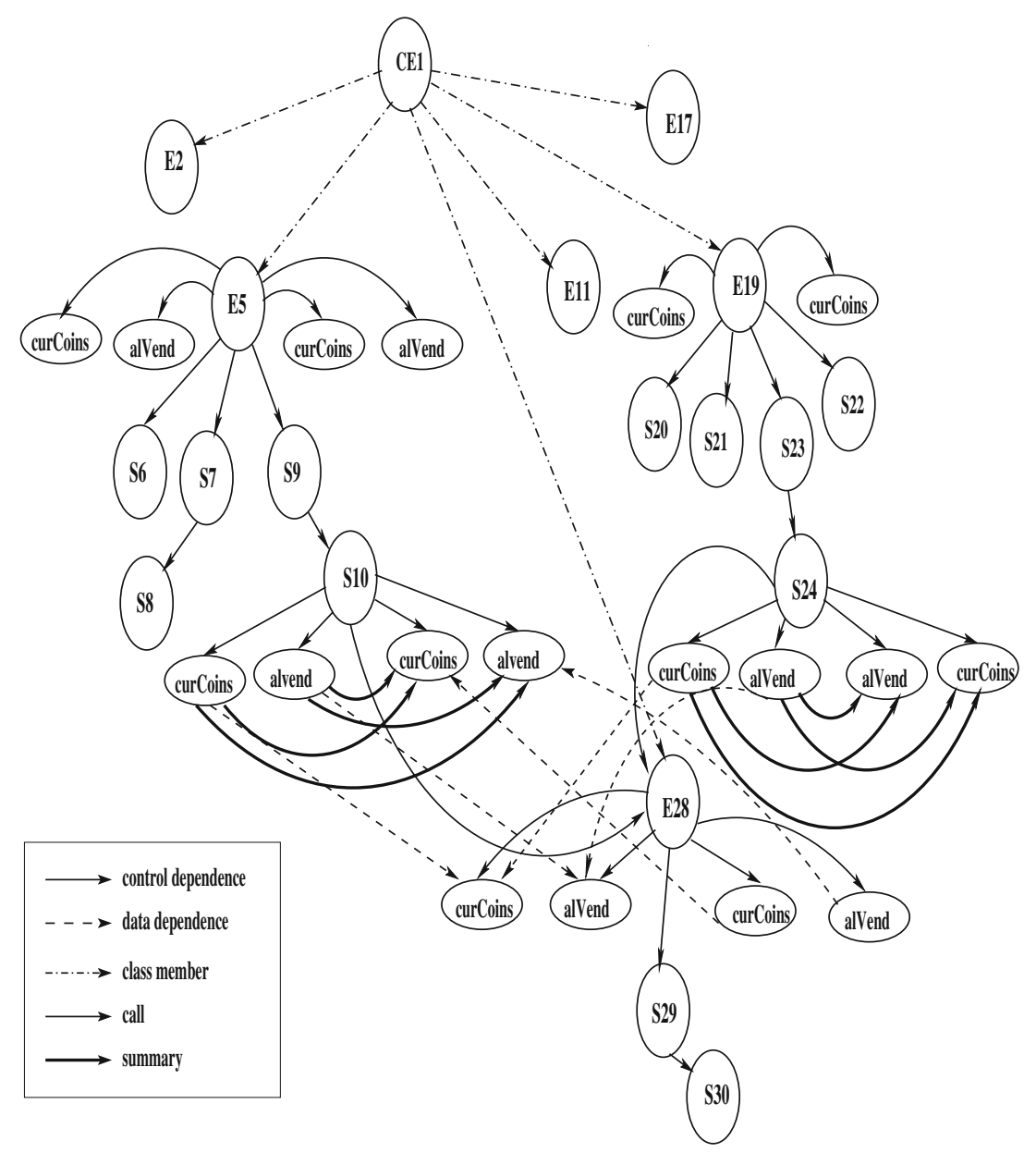


Table 1 Test suite and faults detected

\begin{tabular}{lllllllll}
\hline Test cases & \multicolumn{2}{l}{ Faults } & & & & & & \\
\cline { 2 - 8 } & 1 & 2 & 3 & 4 & 5 & 6 & 7 & 8 \\
\hline$a$ & $\mathrm{X}$ & & & & & & \\
$b$ & $\mathrm{X}$ & & $\mathrm{X}$ & $\mathrm{X}$ & $\mathrm{X}$ & & & \\
$c$ & $\mathrm{X}$ & $\mathrm{X}$ & $\mathrm{X}$ & $\mathrm{X}$ & $\mathrm{X}$ & & & \\
$d$ & & & & $\mathrm{X}$ & & $\mathrm{X}$ & & \\
$e$ & & & & & & $\mathrm{X}$ & $\mathrm{X}$ & $\mathrm{X}$ \\
\hline
\end{tabular}

program contains eight faults and are detected by those test cases, as shown in the Table 1. Consider two ordering of these test cases, order $O_{1}: a-b-c-d-e$, and order $O_{2}: c-$ $e-b-d-a$. Figure $3 \mathrm{a}, \mathrm{b}$ show the percentage of faults detected versus the fraction of the test suite used, for these two orders $O_{1}$ and $O_{2}$ respectively. The area under the curve represents the average of the percentage of faults detected.

In case of test order $O_{1}$, after the execution of test case $a$, one of the eight faults is detected, thus only $12.5 \%$ of faults have been detected after $0.2 \%$ of test cases in test order $O_{1}$ has been used. But, after running test case $b$, four faults are detected and hence $50 \%$ faults have been detected. In contrast, test order $\mathrm{O}_{2}$ detects faults much earlier than test order $O_{1}$. In the test order $O_{2}$, first $0.2 \%$ of test cases detect $62.5 \%$ of the faults and $0.4 \%$ of the test cases in the order detect $100 \%$. Test order $O_{1}$ has an APFD of $50 \%$. As can be observed from Fig. 3, test order $\mathrm{O}_{2}$ detects faults much faster than $O_{1}$, and has an APFD of $70 \%$.

\subsection{Coupling factor (COF) metric}

The COF metric is an object-oriented design model metric from MOOD metric set [24]. The COF metric is used to measure the coupling between classes. The COF metric is calculated using the following formula:
$C O F=\frac{\sum_{i=1}^{T C}\left\lfloor\sum_{j=1}^{T C} i s \_ \text {client }\left(C_{i}, C_{j}\right)\right\rfloor}{T C^{2}-T C}$

where,

is_client $\left(C_{i}, C_{j}\right)= \begin{cases}1 & \text { if }\left(C_{i} \Rightarrow C_{j}\right) \wedge\left(C_{i} \neq C_{j}\right) \\ 0 & \text { otherwise. }\end{cases}$

$C_{i}$ and $C_{j}$ represent the client and supplier class respectively and $T C$ represents total number of classes.

The client-supplier relation, represented by $C_{i} \Rightarrow C_{j}$, means that $C_{i}$ contains at least one non-inheritance reference to a method or attribute of class $C_{j}$. The COF numerator represents the actual number of coupling not imputable to inheritance [25-28].

Coupling relations increase complexity of the system and reduce encapsulation as well as reusability of the system. However, for a given application, classes must cooperate to achieve some kind of functionality.

\section{Related work}

For ordering test cases of procedural programs several approaches have been proposed in the literature based on different criteria. Rothermel et al. developed a family of techniques for TCP based on several structural coverage criteria such as statement coverage, branch coverage, and the probability of exposing known faults [21, 29] etc. Rothermel and coworkers [8] used APFD metric to measure the effectiveness of their techniques. The APFD measure, however, gives better results under two assumptions: all faults are of equal severity, and all test cases have equal costs. Elbaum et al. [8] extended the empirical study reported by Rothermel et al. and additionally considered the coverage criterion at the function level.

The total and the additional coverage strategies are two widely-investigated greedy strategies for test-case prioritization. However, Rothermel et al.'s [21] empirical studies indicate that the greedy strategies may not always produce
Fig. 3 Illustration of APFD measure (a) Test Case Order 01:a-b-c-d-e

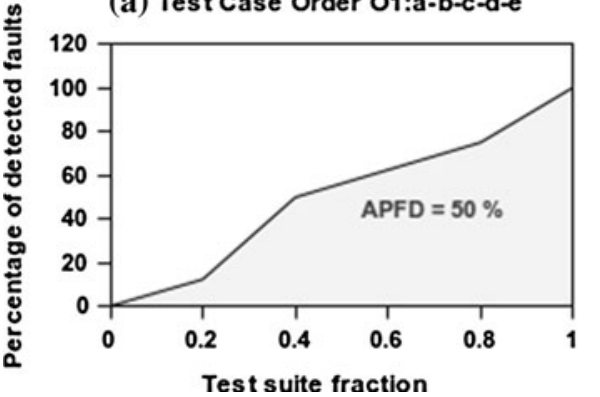

(b) Test Case Order O2: c-e-b-d-a

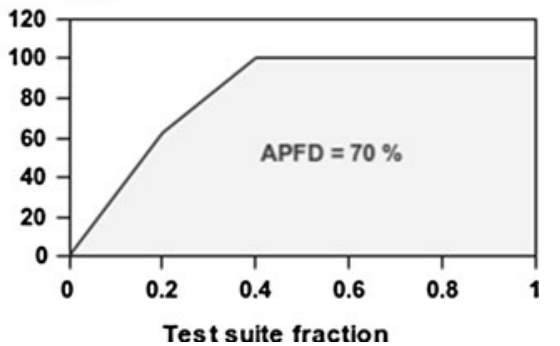


the optimal ordering of test cases. Li et al. [30] further proposed another greedy strategy that is, the two-optimal strategy (based on the k-optimal greedy algorithm [31]) and two meta-heuristic search strategies [32] to prioritize regression test cases.

Statement and function-level TCP techniques are considered in $[8,10,21,22]$ and these do not consider the modified condition/decision coverage (MC/DC) criterion [33]. The MC/DC is a stricter form of decision (or branch) coverage criterion which requires each condition in a decision is to be considered independently in order to reflect its effect on the outcome of the decision.

Elbaum et al. [10, 34] and Park et al. [35] studied the impacts of test costs and fault severities on TCP. Elbaum et al. proposed a cost-cognizant metric to evaluate the effectiveness of their approach called $A P F D_{c}[10,22]$. This metric accounts for varying test case and fault costs during prioritization of regression test cases. In their work, the authors considered functional-level coverage [8] adapted to testing cost and severity level of faults. Park et al. [35] proposed a TCP technique based on estimation of test costs and fault severities that have been obtained using historical information.

Walcott et al. studied the problem of time-aware testcase prioritization and they considered an explicit time budget to carry out regression testing [11]. Walcott et al. used a genetic algorithm-based approach and the authors compared their approach with initial ordering, reverse ordering, random prioritization and fault-aware prioritization techniques.

Another approach for prioritizing regression test cases based on coverage of a relevant slice of the output of a test case was reported by Jeffrey and Gupta [23]. Their goal of prioritizing test cases was to achieve higher rates of fault detection. They defined a relevant slice as the set of statements that influence, or can influence the output of a program when run on a regression test case. Jeffrey and Gupta [23] observed that if a modification to the program affects the output of a regression test case, then it must affect some computation in the relevant slice for that test case. Based on this observation, they prioritized test cases by assigning higher weights to test cases with larger number of statements and branches in its relevant slice, in addition to the statements exercised by it. However, to the best of our knowledge, no work has been reported in the literature on regression TCP of object-oriented programs that considers coverage of various object-oriented features.

\section{S-RTP: our proposed approach}

We have named our proposed approach for regression test prioritization as slice based-regression test prioritization
(S-RTP). Our technique first determines the affected nodes in ESDG model based on an analysis of control and data dependencies as well as dependencies arising from objectrelations and then prioritizes regression test cases based on the number of affected nodes exercised by a test case.

The maintenance phase consists of multiple maintenance cycles as mentioned in Sect. 2.2 There can be many regression testing cycles in each maintenance cycle and in each regression testing cycle, TCP is an important activity.

Figure 4 shows the important steps of our approach carried out in first regression test prioritization cycle using an activity diagram. As shown in Fig. 4, the important activities include constructing the ESDG model, collecting test coverage information and marking the test coverage information on ESDG model and are not repeated for subsequent test prioritization cycles in our approach. We now describe the different activities that are carried out during first regression test cycle in S-RTP in more detail.

- Construct ESDG model: In this step, the ESDG model as described in Sect. 2.1 for the original program $P$ is constructed using a technique similar to that reported in [14].

- Identify changes: The changes between $P$ and the modified program $P^{\prime}$ are identified through semantic analysis and the identified statement-level changes are kept in a file named as differ. Each entry in the differ file contains the changed statements and the line number in both $P$ and $P^{\prime}$. This is shown by the data store Change information in Fig. 4.

- Instrument and execute the program: In this step, $P$ is instrumented by inserting print statements at the basic block level. These print statements serve to collect test coverage information. The instrumented code is executed with the original test suite $T$ to generate information pertaining to the specific statements that are executed for each test case that is the execution trace for each test case. The instrumented statements are stripped during regression test execution, so that the original program behavior is not affected. Generating the test coverage information is an one-time activity for a given program during one testing cycle, and need not be repeated during the subsequent regression testing sessions in that cycle. The test coverage information generated in this step is logged in a file denoted by CovgInfo, and is saved for later processing. This is represented by the data store Test coverage information in Fig. 4.

- Mark ESDG model: In this step, the test coverage information is marked on ESDG model. Marking an ESDG model involves tagging to each node in the ESDG model information about the test cases that execute the corresponding program statement. 
Fig. 4 Activity diagram representation of S-RTP

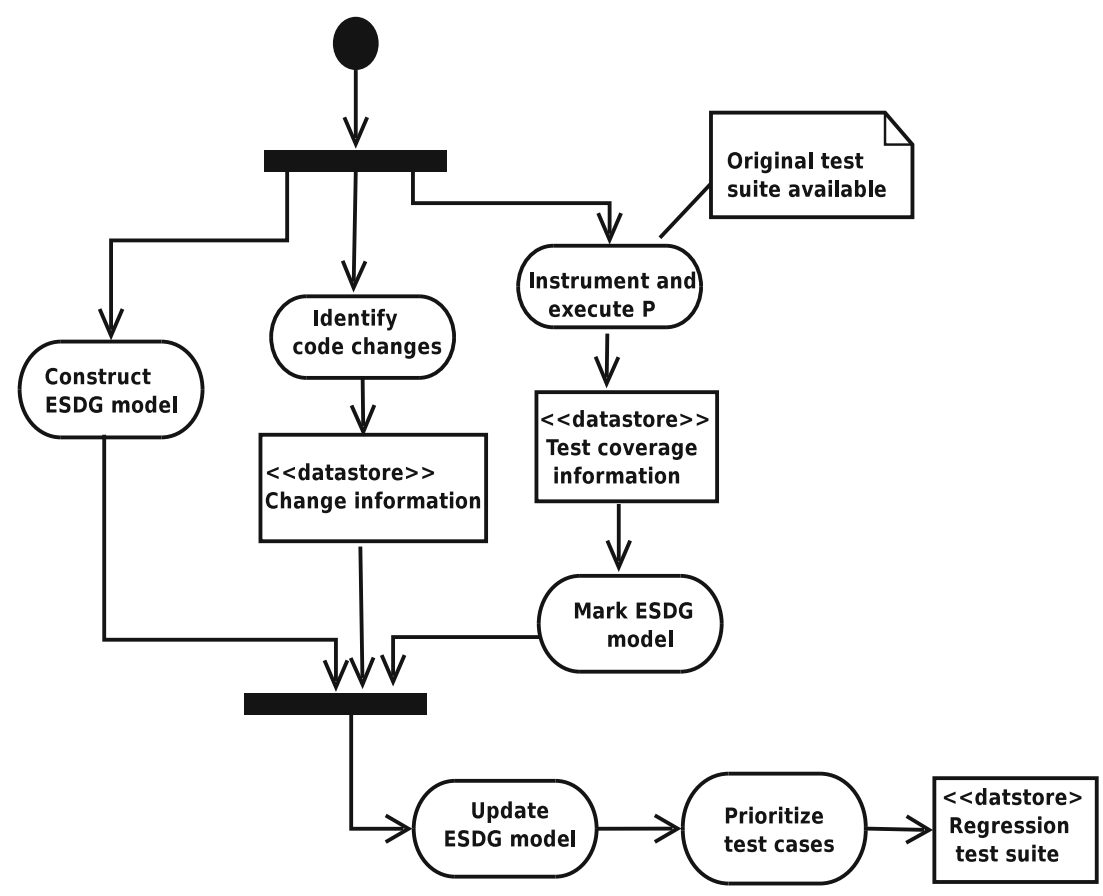

- Update ESDG model: In this step, the model constructed for $P$ is updated using information from differ file during each regression testing cycle to make it correspond to the modified program $P^{\prime}$ and the updated marked ESDG model is denoted by $M_{u}$. A description of the methodology for updation of the ESDG model with change information is discussed in more detail in Sect. 4.2

- Prioritize test cases: In this step, regression test cases are prioritized based on the number of affected nodes exercised by a test case. The prioritized set of regression test cases are represented by the data store Regression test suite in Fig. 4. This step is discussed in more detail in Sect. 4.3

\subsection{Types of program changes}

Without any loss of generality the changes made to a program can be assumed to be one or more of the following types: (1) addition (2) deletion or (3) modification of a statement. A modification operation can be expressed as a deletion operation followed by an addition operation. Hence, we assume that addition and deletion are the two basic types of changes that can be made to a program.

Any change made to a program may affect the dependency relations already existing in the program. Addition of a statement to a program requires creation of a new node and one or more edges in the ESDG model and may also require deletion of some existing edges. This is explained in the following with an example. Consider the code segment shown in Fig. 5a and the corresponding partial ESDG model consisting of control (solid) and data dependence (dotted) edges. In Fig. 5a, $S_{5}$ and $S_{6}$ are two program statements in the method $m$. In the partial ESDG, these two nodes are also denoted by $S_{5}$ and $S_{6}$ and are connected to the method entry node $S_{0}$ by control dependence edges. The partial ESDG model shows that the nodes $S_{5}$ and $S_{6}$ are data dependent on $S_{1}$. Now, suppose we add a statement $S_{j}$ to the original code segment as has been shown in Fig. 5b. After addition of statement $S_{j}$, the statement $S_{6}$ is no longer data dependent on $S_{1}$ and instead is data dependent on statement $S_{j}$. Due to addition of statement $S_{j}$, the data dependence edge between nodes $S_{1}$ and $S_{6}$ needs to be deleted, and two new data dependence edges need to be added between the pairs of nodes $S_{1}$ and $S_{5}$ and $S_{j}$ and $S_{6}$. Also, a control dependence edge from method entry node $S_{0}$ to the node $S_{j}$ is introduced.

Addition or deletion of one or more statements can affect the dependencies existing among program statements, besides those in which the added or deleted statement is directly participate. For example, if a statement defining a variable is deleted, then it would affect the dependency structure of the dependent statements and these statements may have dependency on some other statement(s). So, in case of deletion of a statement, before deleting a statement it is required to identify and mark all those program statements that are data and control dependent or dependent due to object-relations on the deleted statement. Before a node(s) corresponding to the deleted statement is deleted, the other nodes in ESDG model that 
Fig. 5 Effect of addition of a statement on data and control dependencies

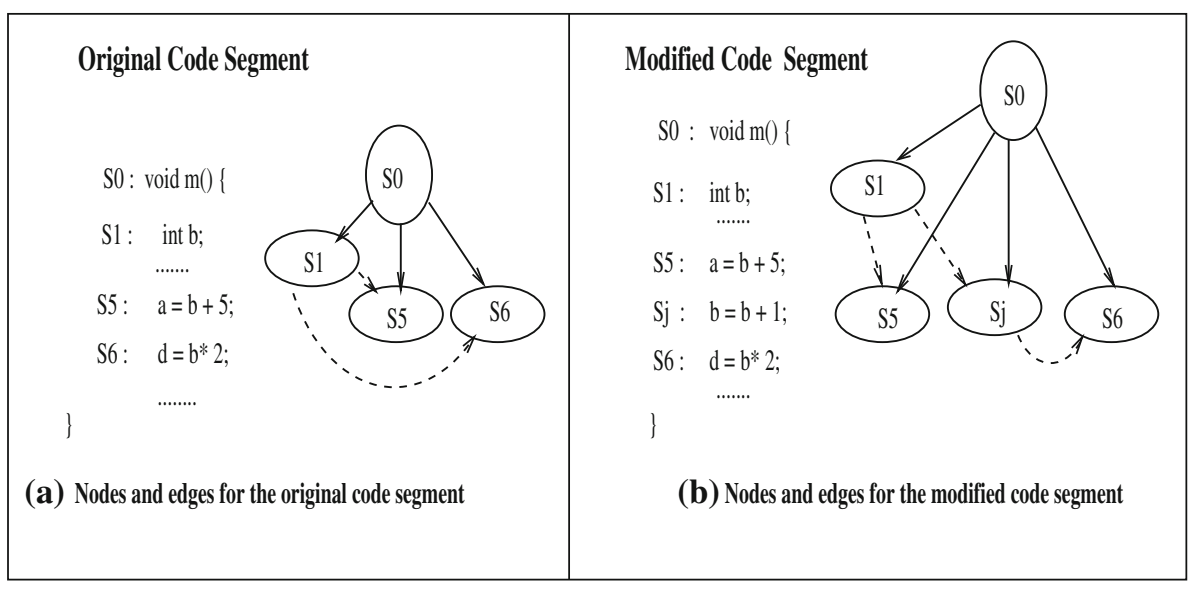

are data or control dependent or dependent due to objectrelations on the deleted node(s) are identified and are marked as affected. Then the node corresponding to the deleted statement is deleted. Subsequently, the in and out edges from the deleted node are also deleted and new dependency edges as required are created.

We give an example of the effect of the deletion of a statement in Fig. 6. Figure 6a shows a code segment and the corresponding partial ESDG model consisting of control (solid) and data dependence (dotted) edges. In the partial ESDG model, the node $S_{q}$ is data dependent on node $S_{p}$ and the node $S_{r}$ is data dependent on nodes $S_{p}$ and $S_{q}$ in the original code. Suppose the statement $S_{q}$ is deleted as shown in Fig. 6b. Before deletion of the node $S_{q}$, the node $S_{r}$ is marked as affected, as the node $S_{r}$ is data dependent on node $S_{q}$. Now, due to deletion of the statement $S_{q}$, an additional data dependence edge is also introduced between the nodes $S_{1}$ and $S_{r}$. Finally, the node $S_{q}$ and all in and out edges of $S_{q}$ are deleted from the partial ESDG model.

\subsection{Updation of an ESDG model}

This section describes our model updation technique by which the constructed ESDG model is updated when a change is made to the corresponding program. We use the term size of the ESDG to mean an upper bound on the number of vertices in an ESDG. Construction of an ESDG model each time a change is made to a program can incur significant overhead especially for large programs. To overcome this problem, instead of creating an ESDG model each time changes are made, we update the original ESDG model to reflect the changes made to it.

For every addition of a statement, usually a single node is created. But for statements such as method call (also called call-site) or called method, more than one node needs to be created in an ESDG model. If it is a method call statement, then in addition to a node for calling method, we create one node for each actual-in and actual-out parameter. The node for calling method is connected to the method entry node by a control dependence edge. Then the call-site node is connected to its method entry node by a call edge. The actual-in parameter nodes are connected to the formal-in parameters nodes in the called method by parameter-in edges. The formal-out node of the called method is connected to the actual-out node by a parameter-out edge. The return statement node of a method is connected to its corresponding method call node using a parameter-out edge. If the value associated with a actual-in
Fig. 6 Effect of deletion of a statement on data and control dependencies

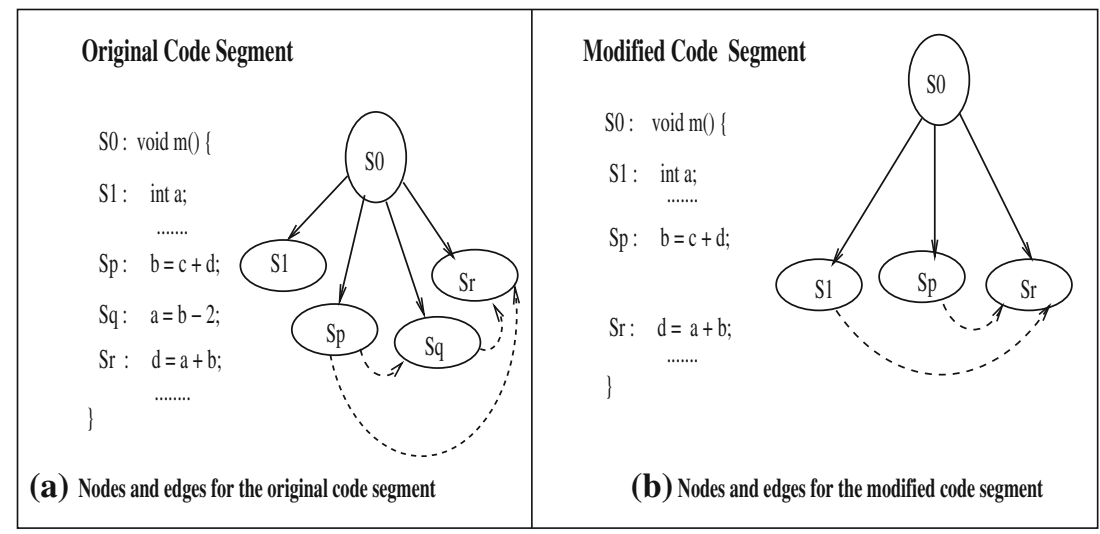




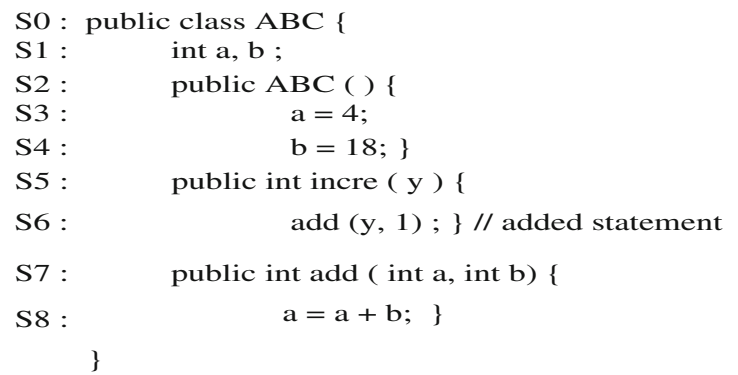

Fig. 7 Example of method call

node affects the value associated with a actual-out node, then a summary edge is added to connect actual-in node to actual-out node. The summary edge represents the transitive dependency across a call-site caused by either control or data dependencies or both and is used for inter-procedural slicing.

Next, we give an example of the effect of addition of a method call statement in the ESDG model. Consider the code segment as shown in Fig. 7. In the code segment, $S_{6}$ is a method call statement. The corresponding partial ESDG model as shown in Fig. 8 represents the addition of different types of nodes and edges due to addition of a method call statement. In the partial ESDG model, square represent the method entry nodes, circle represent statement nodes and ellipses represent the parameter nodes. In Fig. 8, the method call node, method entry node and simple statement nodes are same as statement number in the code segment. The parameter nodes are named according to the parameters passed to the methods as given in the code segment. When the method call statement $S_{6}$ is added in the code segment as shown in Fig. 7, the node $S_{6}$ and the parameter nodes $a \_$in $=y, b \_$in $=1$ and $y=a \_$out are added in the ESDG model. These are connected to $S_{6}$ by control dependence edges. Then $S_{6}$ is connected to the corresponding method entry node $S_{7}$ by a call edge. The actualin parameters that is $a \_i n=y$ and $b \_i n=1$ are connected to the corresponding formal-in parameter nodes $a=a \_i n$ and $b=b \_$in in $S_{7}$ by parameter-in edges. The formal-out parameter node $a \_$out $=a$ of the called method $S_{7}$ is connected to the actual-out node $y=a \_$out by a parameter-out edge. As the actual-in parameter nodes in $S_{6}$ affects the value of actual-out parameter node $y=a_{-}$out, so summary edges are added from $a \_$in $=y$ to $y=a \_$out and b_in $=1$ to $y=a \_o u t$.

For a method entry statement, a method entry node is created and is connected to its class entry node by a class member edge. Similarly, for a simple statement such as assignment, conditional etc., a simple statement node is created, then that simple statement node is connected with its method entry node by a control dependence edge.

In case of deletion of a statement, we delete the corresponding node(or nodes) from ESDG model. But, before deleting the node(or nodes), we determine the nodes that are dependent on the deleted node on account of either control and data dependencies or object-relations such as association or inheritance by slicing, where each deleted node is used as slicing criteria and are marked as affected. After marking these dependent nodes as affected, we delete all in and out edges from the deleted node and then the node corresponding to the deleted statement is deleted from ESDG model.

The changes between the programs $P$ and $P^{\prime}$ are also marked on the ESDG model by using the information from the differ file. This is done as follows: The statements which are deleted from $P$, the nodes in $M$ which were directly data or control dependent on the deleted nodes are affected due to deletion, and are tagged as deleted. These nodes are determined by constructing slices on ESDG model where each deleted node is used as a slicing criterion. Once the model $M$ has been updated to correspond to $P^{\prime}$, for each statement added or modified in $P^{\prime}$, the nodes corresponding to the changed statements in the updated model $M_{u}$ are tagged as changed. We refer to both the set of tagged nodes as Tagged.
Fig. 8 Effect of addition of a method call statement as shown in Fig. 7

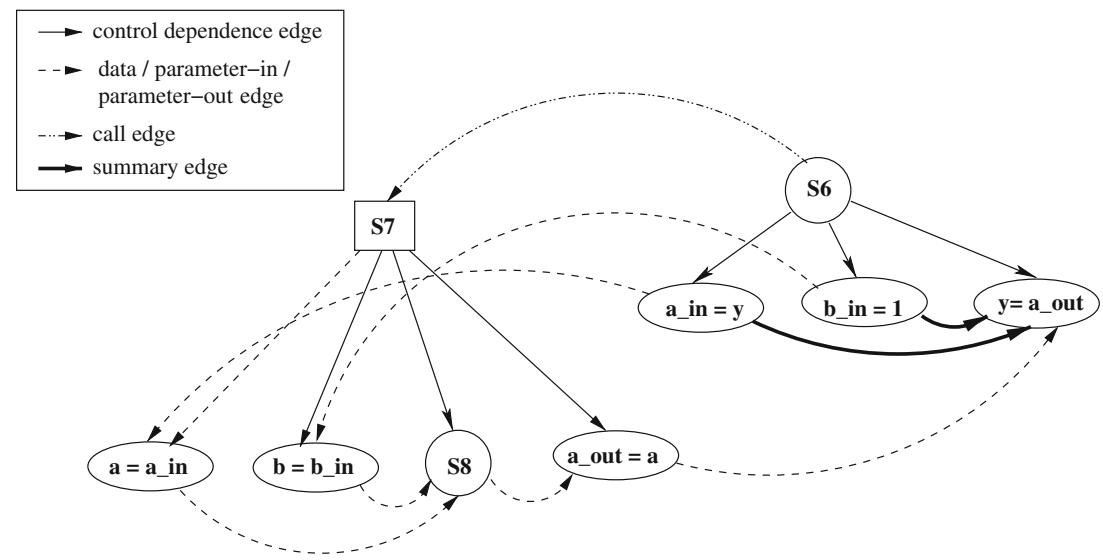




\subsection{TCP using ESDG model}

For prioritization of test cases, first forward slices are constructed on ESDG model by using each added, modified, and affected node as the slicing criterion. Construction of the forward slice helps to determine the nodes that have been affected due to specific modifications made to the code. First all those test cases that exercise the affected model elements are found from forward slice. These are in the regression test suite. Then the test cases are assigned priority based on the number of affected nodes exercised by each selected test case. The pseudocode to determine the affected nodes in ESDG model is given in Algorithm 1. We have named this algorithm ESDGSlice. The working of the algorithm ESDGSlice is described in the following.

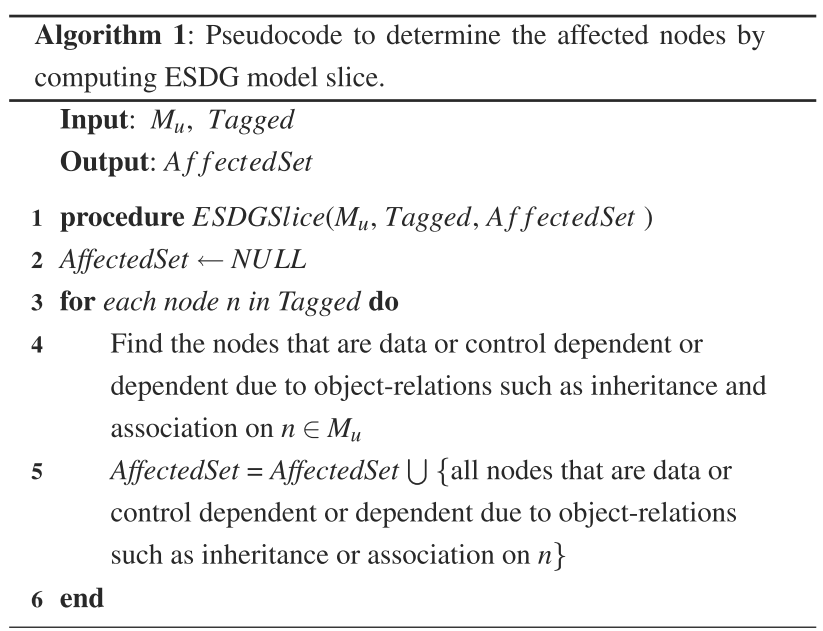

To determine the affected nodes denoted by AffectedSet, we first compute the forward slice on updated marked ESDG model. Our forward slicing algorithm is based on the two-pass graph reachability algorithm proposed by Horwitz et al. [13]. ESDGSlice takes $M_{u}$ and the set of tagged nodes as input, and produces the set of affected nodes denoted by AffectedSet as output. ESDGSlice computes the set of all affected nodes and the steps are given in lines 3-5 in Algorithm 1. Slicing the ESDG model helps to identify the set of model elements that may get affected due to the modifications.

After all the affected nodes in ESDG model are identified through forward slicing, a test case that exercises a higher number of affected nodes is given higher priority. The reason behind it is that, a test case which covers higher number of affected nodes in the dependency model has a more chance to detect an error(s) than a test case which covers lesser number of affected nodes.

Let $T$ be the set of test cases that executes one or more affected nodes in the ESDG model. Initially $T$ is null and let $W T$ be weight for the test cases in $T . W T=$ $\left\{w t_{1}, w t_{2}, \ldots, w t_{n}\right\}$ and $w t_{i}=0$ for all $i$ initially and $n$ represents the $n$th test case in $T$. The pseudocode to prioritize the regression test cases is given in Algorithm 2. The algorithm to prioritize test cases is named as TestPrior and the input to TestPrior is the $M_{u}, W T$ and the AffectedSet. The output of TestPrior is the prioritized set of regression test cases denoted by $T$. To prioritize test cases, for each node $n_{i}$ in AffectedSet, add the test case $t_{j}$ executing $n_{i}$ to $T$ and the weight of $t_{j}$ denoted by $w t_{j}$ is increased by 1 . Step 4 in Algorithm 2 is trivial since the information about which test cases execute which model elements is already marked on the corresponding node in the ESDG model. Finally, the test cases in $T$ are sorted according to their decreasing weights in the list $W T$.

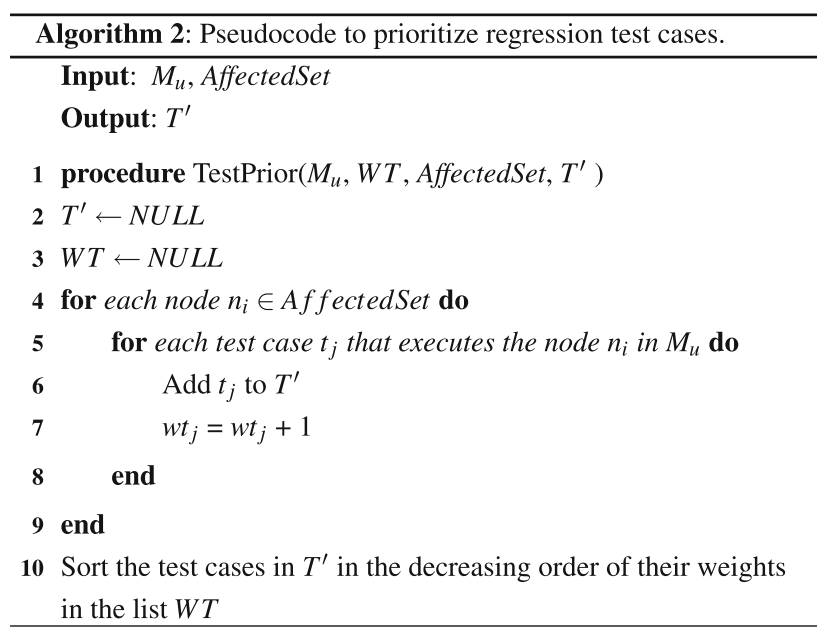

\section{Experimental study}

In this Section, we first discuss a prototype tool Regression-TEST Prioritization (R-TESTPrio) that we have developed to implement our approach. Next, we explain the experimental setups made and the results achieved in the subsequent subsections.

In order to measure the effectiveness of our approach, we have used the APFD metric [21]. In our experimental study, we first prioritize the test cases using S-RTP. Then APFD values are calculated for the prioritized test suite of each of the considered program. We have compared our approach with Smith et al.'s approach [36]. We have implemented their approach only for non-overlap-aware greedy algorithm [37] to prioritize test cases. For their approach, we build a calling context tree (CCT) for a program as described in [38]. The call tree constructor uses instrumentation probes to create the CCT. After the call tree is initialized, the tree constructor executes a probe before and after the execution of each method and test case. 
We prioritize the test cases using Smith et al.'s approach based on coverage of call tree paths by a test case and calculate the APFD metric for Smith et al.'s approach [36] for the prioritized test suite. In order to evaluate the effectiveness of our approach, we compare the APFD values for each of the considered program as calculated using S-RTP and Smith et al.'s approach.

\subsection{R-TESTPrio: a prototype implementation of S-RTP}

R-TESTPrio has been developed using the programming language Java on a Microsoft Windows XP environment. The code size of R-TESTPrio is approximately 14,000 LOC, excluding the external packages that are used in implementation of S-RTP technique. The user interface of $\mathrm{R}$-TESTPrio is developed using Java Swing.

We now briefly mention the various open source software packages used to implement our approach and the different components of R-TESTPrio in the following.

Open source software packages used: The tool R-TESTPrio is developed using the following open source software packages: Eclipse [39], ANTLR [40] and Graphviz [41]. We have used eclipse as an IDE and ANTLR as the parser generator, and have adapted ANTLR grammar file for Java language [42]. ANTLR works as an Eclipse plugin. We have used Graphviz to graphically present the ESDG model constructed by R-TESTPrio. We have used an Eclipse plug-in version of an open source software $\mu \mathrm{Java}$ (MuJava), a mutation system for Java programs to generate mutants [43]. The eclipse plug in version of MuJava is known as MuClipse. (An open source mutation testing plugin for Eclipse http://muclipse.sourceforge.net/). It provides a bridge between the MuJava API and Eclipse. MuClipse automatically generates mutants for both traditional and class-level mutation operators.

Components of R-TESTPrio: Figure 9 shows a schematic representation of our prototype tool. R-TEST-Prio comprises of 5 major components, namely, ESDG Model Constructor, Test Coverage Generator, ESDG Model Marker, ESDG Model Updater and Test Case Prioritizer. In Fig. 9, rectangles represent the different components of R-TESTPrio and the ellipses represent data or artifacts. Initially, R-TESTPrio takes $P, P^{\prime}$, Change Information and the initial test suite $T$ as input. In R-TESTPrio, the input to a component is used as an output of an another component. The component Test Coverage Generator produces output Test Coverage Information that is used as an input to the component ESDG Model Marker in R-TESTPrio. The Change Information (described in Sect. 4) is used as an input to the component ESDG Model Updater to update the original model $M$.

We describe the working of different components of R-TESTPrio in the following.

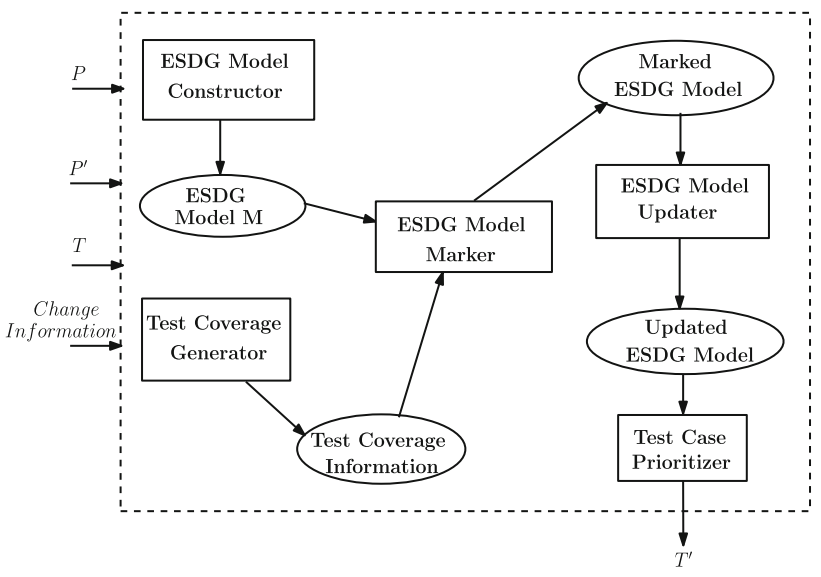

Fig. 9 Schematic of design of R-TESTPrio

- ESDG Model Constructor: ESDG Model Constructor is developed in Java using Eclipse IDE [39] and ANTLR tool [40]. ESDG model constructor takes as input a Java program and constructs the ESDG model for the program.

- Test Coverage Generator: This component generates test coverage information by executing the input program with all test cases from $T$. The generated test coverage information is stored in file CovgInfo that lists the line numbers executed by each test case in $T$.

- ESDG Model Marker: This component of R-TESTPrio stores the test coverage information from CovgInfo in the ESDG model. This approach of storing test coverage information in the model itself circumvents the use of file storage and improves the efficiency of our approach. To store the test coverage information, ESDG model is traversed to find out the corresponding node(s) modeling each program statement $s$ in $P$. Then, for each node corresponding to a program statement $s$, ESDG Model Marker stores the list of test cases that execute the node corresponding to $s$.

- ESDG Model Updater: ESDG Model Updater component is used to update the ESDG model. This component takes the differ file as external input. The updated model reflects the changes that are made to $P$.

- Test Case Prioritizer: This component prioritizes the test cases based on the number of affected nodes exercised by different test cases. The output of this component is a file that contains the identifiers of regression test cases in priority order.

\subsection{Experimental setup}

We have used seven Java programs in our experimentation: elevator control (EC), cruise control $(\mathrm{CrC})$, binary search tree (BST), automated teller machine (ATM), power window controller (PWC), ordered set (OrS) and vending 
machine (VM). The characteristics of these programs have been given in Table 2 .

The sizes of the considered programs ranged from 229 to 943 lines of source code and the sizes of the test suites ranged from 17 to 42 test cases. The first column in Table 2 shows the programs that were used in the experimental studies. Columns 2 and 3 show the sizes of the programs and the number of classes for our example programs respectively. The total number of test cases in each of the considered program is given in column 4 and the total number of mutation faults of each program in column 5 . The test cases for these considered programs were designed by us using black-box test case design techniques.

The programs that we have considered in our study were of moderate size and faults were generated through code mutation using MuClipse (An open source mutation testing plug-in for Eclipse http://muclipse.sourceforge.net/). MuClipse provides an API to generate mutants. It allows one to select the types of mutation operators to be employed. MuClipse uses two types of mutation operators, class level and method level. Class level operators are specialized to object-oriented faults, whereas the method level operators are the same as traditional mutation operators. The specific class level and the traditional operators that are supported by MuClipse are discussed in [44-47] respectively.

We have used COF metric to determine the interdependencies between classes and COF metric is described in more detail in Sect. 2.5 The programs having higher COF value indicates higher interdependency. We have categorized our considered programs into 3 types, that is, low, moderate and high based on this aspect and is shown in Table 3. The programs having COF value up to 1 are considered as low, more than 1 and up to 2 as moderate and more than 2 as high in our approach. In Table 3, column 1 represents the program names, columns 2 and 3 represent the COF value for each program and the category of programs respectively.

\subsection{Results}

In this section, we present the results obtained from our experimental studies. Table 4 summarizes the main results.

Table 2 Summary of programs used in experimentation

\begin{tabular}{lllll}
\hline $\begin{array}{l}\text { Program } \\
\text { name }\end{array}$ & Size (LOCs) & Classes & Test cases & $\begin{array}{l}\text { Mutation } \\
\text { faults }\end{array}$ \\
\hline EC & 943 & 8 & 18 & 72 \\
CrC & 649 & 4 & 42 & 46 \\
BST & 564 & 4 & 17 & 31 \\
ATM & 623 & 3 & 25 & 38 \\
PWC & 720 & 6 & 36 & 81 \\
OrS & 229 & 2 & 18 & 42 \\
VM & 471 & 3 & 25 & 53 \\
\hline
\end{tabular}

Table 3 Categories of programs

\begin{tabular}{lll}
\hline Program name & COF & Program categories \\
\hline BST & 0.58 & \\
ATM & 0.83 & Low \\
OrS & 0.5 & \\
EC & 1.06 & Moderate \\
VM & 1.8 & \\
CrC & 2.2 & High \\
PWC & 2.5 & \\
\hline
\end{tabular}

The columns 2 and 3 of Table 4 show the APFD for R-TESTPrio and Smith et al.'s approach in percentage for each of our considered programs respectively and column 4 represents the percentage increase in APFD for R-TESTPrio over that of Smith et al.'s approach.

From Table 4 it can be observed that for all the considered programs, APFD of R-TESTPrio is on the average $25.70 \%$ higher as compared to Smith et al.'s approach. This increase may be due to the fact that, our approach considers several intrinsic features of an object-oriented program such as class, dynamic binding and object-relations like inheritance, association and polymorphism for prioritization.

The results of Table 4 have been presented in the form of a bar graph in Fig. 10. In Fig. 10, the $x$-axis represents the programs used in our experiments and $y$-axis represents the APFD values for all programs using S-RTP and Smith et al.'s approach.

For the considered programs, we have also analyzed the behavior of the program with increase in the number of mutation faults. For analysis of individual program, we choose one program from each considered category as given in Table 3 in our experimental studies. We have considered the programs $\mathrm{CrC}$, EC and BST with high, moderate and low COF values respectively. We calculate the APFD values of each of the program with increase in the number of mutation faults and analyze the trend in APFD values for different categories of programs.

For each of the considered programs $\mathrm{CrC}, \mathrm{EC}$ and BST, we determine the APFD values using R-TESTPrio. We

Table 4 Summary of experimental results

\begin{tabular}{llll}
\hline $\begin{array}{l}\text { Program } \\
\text { name }\end{array}$ & $\begin{array}{l}\text { APFD for } \\
\text { R-TESTPrio }\end{array}$ & $\begin{array}{l}\text { APFD for Smith et al.'s } \\
\text { [36] approach }\end{array}$ & $\begin{array}{l}\% \\
\text { Increase }\end{array}$ \\
\hline EC & 86.2 & 68.6 & 25.65 \\
CrC & 79.6 & 60.4 & 28.01 \\
BST & 83.4 & 64.1 & 30.10 \\
ATM & 81.1 & 63.7 & 27.31 \\
PWC & 82.2 & 66.7 & 23.23 \\
OrS & 85.2 & 68.7 & 24.01 \\
VM & 80.4 & 66.1 & 21.63 \\
\hline
\end{tabular}




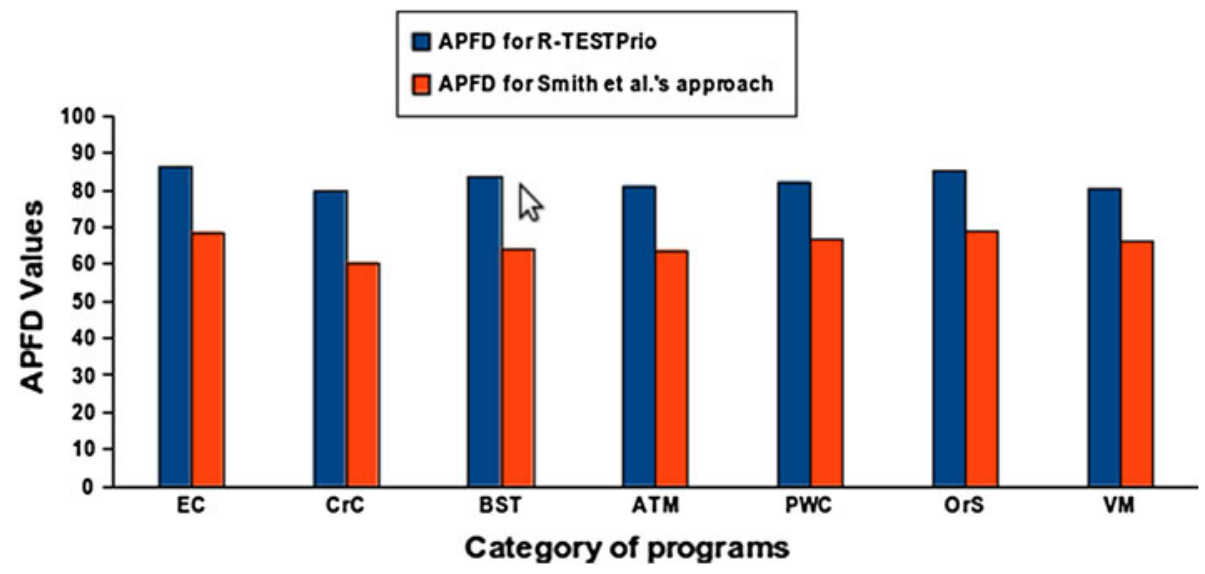

Fig. 10 Comparison of APFD values for S-RTP and Smith et al.'s approach

increase the number of mutation faults from $5 \%$ to $25 \%$ for all three considered programs. The Tables 5, 6 and 7 show the results obtained from our experimental study for the analysis of the specific programs $\mathrm{CrC}, \mathrm{EC}$ and BST. In

Table 5 APFD values for the program cruise controller using R-TESTPrio

\begin{tabular}{lll}
\hline Program name & \% of mutation faults & APFD for R-TESTPrio \\
\hline CrC & 5 & 38.3 \\
& 10 & 39.5 \\
& 15 & 42.7 \\
& 20 & 49.1 \\
& 25 & 60.3 \\
\hline
\end{tabular}

Table 6 APFD values for the program elevator controller using R-TESTPrio

\begin{tabular}{lcl}
\hline Program name & \% of mutation faults & APFD for R-TESTPrio \\
\hline EC & 5 & 15.2 \\
& 10 & 16.1 \\
& 15 & 17.4 \\
& 20 & 20.4 \\
& 25 & 23.9 \\
\hline
\end{tabular}

Table 7 APFD values for the program binary search tree using R-TESTPrio

\begin{tabular}{lll}
\hline Program name & $\%$ of mutation faults & APFD for R-TESTPrio \\
\hline BST & 5 & 21.5 \\
& 10 & 22.7 \\
& 15 & 24.3 \\
& 20 & 26.5 \\
& 25 & 30.2 \\
\hline
\end{tabular}

the Tables 5, 6 and 7, the first column represents the program, column 2 represents the \% increase in the number of mutation faults, and column 3 shows the APFD values in percentage while using R-TESTPrio and increasing the number of mutation faults.

The results Tables 5, 6 and 7 have been presented in the form of line graphs in Figs. 11, 12 and 13 respectively. In

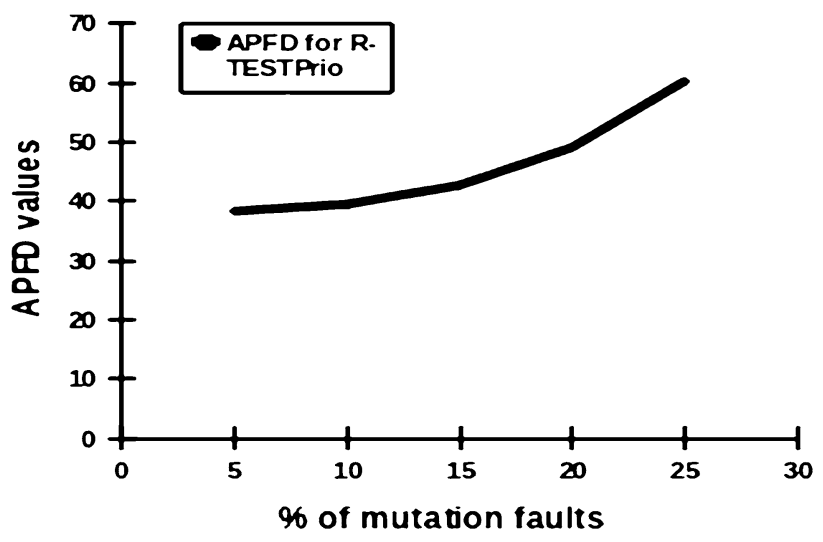

Fig. 11 APFD values for the program $\mathrm{CrC}$

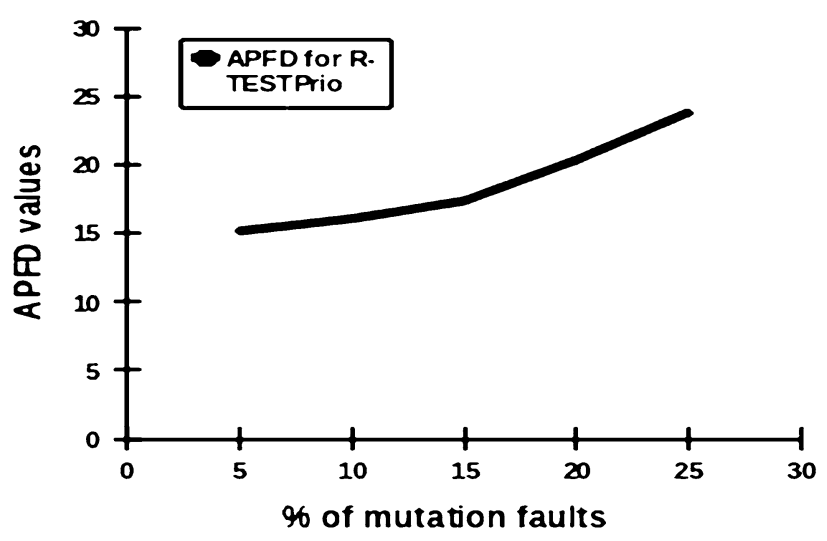

Fig. 12 APFD values for the program EC 


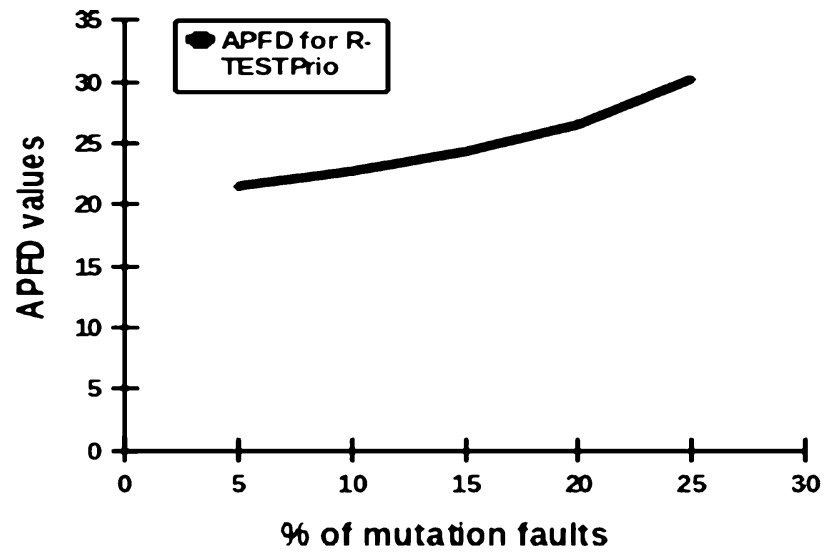

Fig. 13 APFD values for the program BST

these graphs, $x$-axis represents the $\%$ of mutation faults and $y$-axis represents the APFD values for the programs using R-TESTPrio.

From Tables 5, 6 and 7 it can be observed that, for all the three categories of programs, with increase in the number of mutation faults, APFD values using R-TESTPrio increases. This increase may be due to the fact that, with increase in the number of faults, the prioritization order using R-TESTPrio increases the rate of fault detection. But, the percentage increase in the APFD values with increase in the number of mutation faults is higher for $\mathrm{CrC}$ as compared to EC and BST and EC is higher as compared to BST. This may be due to the fact that, with increase in the interdependency between classes, the percentage increase in the APFD values for the programs with high interdependency is higher as compared to the programs with low interdependency.

\section{Comparison with related work}

Literature study indicate that, most of the proposed regression TCP techniques order test cases based on greedy strategies using coverage criteria such as statement coverage, additional statement coverage, and function coverage etc. for procedural programs. But, these techniques are not suitable for object-oriented programs as statement and function coverage are not very meaningful due to objectoriented features such as inheritance and dynamic binding [12]. According to Thuy, exercising a single binding of a polymorphic call by a test case is not sufficient and it is required to exercise all possible bindings [48]. Further, a regression test prioritization technique for object-oriented programs needs to consider dependencies introduced by various object-relations.

A regression test reduction and prioritization approach using call trees was proposed in the literature for object- oriented programs by Smith et al. [36]. To the best of our knowledge no directly comparable work on regression TCP of object-oriented programs based on coverage of objectoriented features has been reported in the literature. Consequently, we have compared our approach with Smith et al.'s approach. The results of our experimental study indicate that, the APFD metric values for R-TESTPrio is on an average higher by about $25.70 \%$ over Smith et al.'s approach.

\section{Conclusion}

We have proposed an approach for regression TCP that orders test cases based on the number of affected model elements exercised by a test case in the program model of object-oriented programs. We determine the affected parts of the program based on consideration of dependencies arising on account of object-relations in addition to the data and control dependencies. For this, we construct an ESDG model and the affected elements are determined based on slicing the ESDG model. Our approach can be generalized to consider other object-oriented programming features such as exceptions, threads, interfaces and class libraries by suitably augmenting the graph model. We have evaluated the effectiveness of our proposed regression TCP technique using several moderate sized Java programs. The results of our study show that our approach on an average achieves an increase in the APFD value by $25.70 \%$ compared to a related approach.

\section{References}

1. Harrold M, Jones J, Li T, Liang D, Orso A (2001) Regression test selection for java software. In: Proceedings of the 16th ACM SIGPLAN conference on object-oriented programming, systems, languages and applications, pp 312-326

2. Rothermel G, Harrold M, Dedhia J (2000) Regression test selection for C++ software. J Softw Test Verif Reliab 10(6):77-109

3. Rothermel G, Harrold MJ (1994) Selecting regression tests for object-oriented software. In: Proceedings of the International Conference on Software Maintenance, Victoria, pp 14-25

4. Harrold M, Gupta R, Soffa M (1993) A methodology for controlling the size of a test suite. ACM Trans Softw Eng Methodol 2(3):270-285

5. Korel B, Tahat L, Vaysburg B (2002) Model based regression test reduction using dependence analysis. In: Proceedings of IEEE International Conference on Software Maintenance, pp 214-223

6. Ma X, Sheng B, Ye C (2005) Advanced parallel processing technologies, chapter test-suite reduction using genetic algorithm. Springer, Berlin

7. Wong WE, Horgan JR, London S, Mathur AP (1998) Effect of test set minimization on fault detection effectiveness. Softw Pract Experience 28(4):347-369

8. Elbaum S, Malishevsky A, Rothermel G (2002) Test case prioritization: a family of empirical studies. IEEE Trans Softw Eng 28(2):159-182 
9. Elbaum S, Rothermel G, Kanduri S, Malishevsky A (2004) Selecting a cost-effective test case prioritization technique. Softw Qual Control 12(3):185-210

10. Malishevsky A, Ruthruff J, Rothermel G, Elbaum S (2006) Costcognizant test case prioritization. Technical Report TR-UNLCSE-2006-0004

11. Walcott K, Soffa M, Kapfhammer G, Roos R (2006) Time-aware test suite prioritization. In: Proceedings of the 2006 International Symposium on Software Testing and Analysis, pp 1-12

12. Binder RV (2003) Testing object-oriented systems: models, patterns, and tools. Addison-Wesley, Menlo Park

13. Horwitz S, Reps T, Binkley D (1990) Interprocedural slicing using dependence graphs. Trans Program Lang Syst 12(1):26-60

14. Larsen L, Harrold M (1996) Slicing object-oriented software. In: Proceedings of 18th IEEE International Conference on Software Engineering

15. Ferrante J, Ottenstein KJ, Warren JD (1987) The program dependence graph and its use in optimization. ACM Trans Program Lang Syst 9(3):319-349

16. Website. http://www.bugzilla.org/. Accessed 30 July 2012

17. Weiser M (1981) Program slicing. In: ICSE'81: Proceedings of the 5th International Conference on Software Engineering, pp 439-449

18. Tip F (1995) A survey of program slicing techniques. J Program Lang 3:121-189

19. Gallagher K, Lyle J (1991) Using program slicing in software maintenance. IEEE Trans Softw Eng 17(8):751-761

20. Mund G, Mall R (2006) An efficient interprocedural dynamic slicing method. J Syst Softw 79(6):791-806

21. Rothermel G, Untch R, Chu C, Harrold M (2001) Prioritizing test cases for regression testing. IEEE Trans Softw Eng 27(10): 929-948

22. Elbaum S, Malishevsky A, Rothermel G (2001) Incorporating varying test costs and fault severities into test case prioritization. In: Proceedings of the 23rd International Conference on Software Engineering, pp 329-338, Ontario

23. Jeffrey D, Gupta N (2008) Experiments with test case prioritization using relevant slices. J Syst Softw 81:196-221

24. Brito e Abreu F (1995) The MOOD metrics set. In: Proceedings of ECOOP'95 Workshop on Metrics

25. Abreu F, Carapuca R (1994) Object-oriented software engineering: measuring and controlling the development process. In: Proceedings of the 4th International Conference on Software Quality, McLean, pp 231-247

26. Abreu F, Goulao M, Esteves R (1995) Toward the design quality evaluation of object-oriented software systems. In: Proceedings of the 5th International Conference on Software Quality, Austin

27. Abreu F, Melo W (1996) Evaluating the impact of object-oriented design on software quality. In: Proceedings of the 3rd International Software Metrics Symposium (METRICS'96). IEEE, Berlin, pp 90-99

28. Harrison R, Counsell S, Nithi R (1998) An evaluation of the MOOD set of object-oriented software metrics. JIEEE Trans Softw Eng 24(6):491-496
29. Rothermel G, Untch RH, Chu C, Harrold MJ (1999) Test case prioritization: an empirical study. In: Proceedings of ICSM, pp $179-188$

30. Li Z, Harman M, Hierons R (2007) Search algorithms for regression test case prioritization. IEEE Trans Softw Eng 33(4):225-237

31. Lin S (1965) Computer solutions of the travelling salesman problem. Bell Syst Tech J 44(5):2245-2269

32. Reeves CR (1993) Modern heuristic techniques for combinatorial problems. Wiley. New York

33. Jones JA, Harrold MJ (2003) Test-suite reduction and prioritization for modified condition/decision coverage. IEEE Trans Softw Eng 29(3):195-209

34. Elbaum S, Malishevsky A, Rothermel G (2000) Prioritizing test cases for regression testing. In: Proceedings of ISSTA, ACM Press, pp 102-112

35. Park H, Ryu H, Baik J (2008) Historical value-based approach for cost-cognizant test case prioritization to improve the effectiveness of regression testing. In: Proceedings of SSIRI, pp 39-46

36. Smith A, Geiger J, Kapfhammer GM, Soffa ML (2007) Test suite reduction and prioritization with call trees. In: Proceedings of ASE'07, Atlanta, 4-9 Nov 2007

37. Rummel M, Kapfhammer GM, Thall A (2005) Towards the prioritization of regression test suite with data flow information. In: Proceedings of 20th SAC, pp 1499-1504

38. McMaster S, Memon A (2005) Call stack coverage for test suite reduction. In: Proceedings of 21st ICSM, pp 539-548

39. Eclipse. http://www.eclipse.org/. Accessed 16 May 2012

40. Antlr Parser Generator. http://www.antlr.org/. Accessed 14 May 2012

41. Graphviz (2012). http://www.graphviz.org/. Accessed 21 Aug 2012

42. Scott Wisniewski. ANTLR Java Grammar. http://www.antlr.org/ grammar/list. Accessed 14 May 2012

43. Ma Y-S, Offutt J, Kwon Y-R (2005) MuJava: an automated class mutation system. J Softw Test Verif Reliab 15(2):97-133

44. Kim S, Clark J, McDermid J (2000) Class mutation: mutation testing for object-oriented programs. OOSS: Object-Oriented Software Systems

45. Ma Y-S, Kwon Y-R, Offutt J (2002) Inter-class mutation operators for Java. In: Proceedings of the 13th International Symposium on Software Reliability Engineering. IEEE Computer Society Press, Annapolis, pp 352-363

46. Agrawal H, DeMillo RA, Hathaway R, Hsu W, Wynne H, Krauser EW, Martin RJ, Spafford EH (1989) Design of mutant operators for the $\mathrm{C}$ programming language. In: Technical Report: SERC-TR-41-P, Software Engineering Research Center, Purdue University

47. Delamaro ME, Maldonado JC, Mathur AP (2001) Interface mutation: an approach to integration testing. IEEE Trans Softw Eng 27(3):228-247

48. Thuy NN (1992) Testability and unit tests in large object oriented software. In: Proceedings, 5th International Software Quality Week. Software Research, San Francisco 